\title{
Interaction between Self-Care and Caregiving Ability on Home Discharged Stroke Patients Based on Motor Functional Independence Measure Score in Recovery Ward
}

\author{
Takashi Kimura ${ }^{1,2}$ \\ ${ }^{1}$ Department of Physical Therapy, ASO Rehabilitation College, Fukuoka, Japan \\ ${ }^{2}$ Department of Rehabilitation Medicine, Saga University Hospital, Saga, Japan \\ Email: t.kimufuku@gmail.com
}

How to cite this paper: Kimura, T. (2021) Interaction between Self-Care and Caregiving Ability on Home Discharged Stroke Patients Based on Motor Functional Independence Measure Score in Recovery Ward. Open Journal of Therapy and Rehabilitation, 9, 42-56.

https://doi.org/10.4236/ojtr.2021.92004

Received: February 7, 2021

Accepted: March 29, 2021

Published: April 1, 2021

Copyright $\odot 2021$ by author(s) and Scientific Research Publishing Inc. This work is licensed under the Creative Commons Attribution International License (CC BY 4.0).

http://creativecommons.org/licenses/by/4.0/

\begin{abstract}
Background: A correlation has been found between self-care and caregiving ability in home discharged stroke patients. However, few studies have explored the interaction between individual self-care and caregiving ability. Purpose: This study aimed to investigate the interaction between individual self-care and caregiving ability in home discharged stroke patients. Participants and Methods: A total of 2688 stroke patients registered in the Japanese Rehabilitation Database (JRD) were retrospectively analyzed. Patients were classified into two groups based on their discharge destination (home discharge, non-home discharge), and the data were modified as mean-centered values. After a correlation analysis was performed between all the collected data, a hierarchical multiple regression analysis evaluated the interaction using discharge destination as the dependent variable. Model 1 used two independent variables (Rankin scale and cognitive functional independence measure score), model 2 used two independent variables (caregiving ability and individual self-care), and model 3 used a mean-centering value, which was added to model 2. A simple slope was used for further analysis. Results: Self-care showed an interaction with caregiving ability. The $\mathrm{R}^{2}$ changes in models 1 and 2 were significant in the following: grooming, bathing, gait, and climbing stairs $(\mathrm{p}<0.01)$. Additionally, the results of the simple slope analysis were significant for all areas. Conclusion: Based on the JRD, this study suggested that a focus on improving these areas, especially climbing stairs, is effective in preparing for home discharge and should be positively considered for stroke patients.
\end{abstract}




\section{Keywords}

Interaction, Stroke, ADL, FIM, Recovery Ward

\section{Introduction}

Stroke causes various long-term symptoms and post-stroke disabilities. Stroke patients have functional disabilities, of which gait and activities of daily living (ADL) disturbances are the most common consequences [1] [2]. For these reasons, more than half of stroke patients need to be partially or totally assisted by others in ADL after discharge [3] [4]. Stroke patients' caregivers experience various problems ranging from physical, mental, social to economic problems that affect their quality of life [4] [5]. Therefore, it is necessary to address not only physical but also environmental factors such as human resources that affect stroke patients living at home [6]. However, comprehensively considering and adjusting these factors in an aging society with a decreasing birth-rate is difficult. Consequently, it tends to be more difficult to return home after getting discharged from the hospital, even if they have such hope at admission. Moreover, stroke is one of the major causes of loss of independence and lower quality of life. Additionally, stroke has been cited as one of the major causes of long-term caregiving. Hence, the aim of rehabilitation on recovery is to enhance the ADL in stroke patients. Thus, it is necessary to effectively improve ADL based on prognosis prediction in the early stage and planning after leaving the hospital. However, discharge planning is difficult to undertake while comprehensively considering the various factors surrounding stroke patients, and there is a chance that they may not be able to return to their home after leaving the hospital even if they hoped to do so at admission. Previous studies have investigated reports on factors affecting home discharge and a prediction using outcomes [7] [8]. These papers showed a relationship between ADL, Functional Independence Measure (FIM), and home discharge [9] [10] [11] [12]. Moreover, the number of cohabitating people is an important factor for caregiving at home and a crucial condition for home discharge, as many studies have shown a significant [11] [12] [13]. However, despite the extensive literature available on the relationship between ADL, caregiving ability, and home discharge, the interaction between caregiving ability and improvements in individual ADL has not been sufficiently elucidated in stroke patients. Interventions related to ADL could differ depending on the existence of an interactive relationship between ADL and caregiving ability. Thus, the interaction between ADL and caregiving ability could lead to a more significant possibility of home discharge. This could be beneficial for improving quality of life through ADL training during the rehabilitation of stroke patients. Therefore, it is important to clarify the existence of an interaction between caregiving ability and ADL.

This study aimed to investigate the interaction between caregiving ability and 
individual self-care activities for home discharge based on the motor-FIM score.

\section{Materials and Method}

\subsection{Subjects}

The medical data of 6875 patients with stroke were extracted from the Japan Rehabilitation Database (JRD) in the stroke/recovery rehabilitation phase ward (January 2016 version, https://square.umin.ac.jp/JARD/). We used anonymized observational data obtained in normal clinical settings. The need for informed consent was waived due to the retrospective and observational design of the study and the use of secondary data. The original data collection had been approved by the Ethics Committee of the Japanese Association of Rehabilitation Medicine (November 14, 2014). However, the committee did not issue an approval number. A flowchart of the subject in this study is shown in Figure 1. The inclusion criteria were as follows: age, 20 - 100 years; days from onset to admission, 20 - 200 days; and length of ward stay, 1 - 255 days. Similarly, all data were collected based on FIM at discharge, Rankin scale (RS) at discharge, the destination of leaving hospital excepting incompletely filed. The exclusion criteria were death, changing hospital due to sudden turn for the worse and individuals who lived in nursing homes or other residential facilities prior to stroke onset and finally, the data of 2688 cases were analyzed. The FIM score is reported to have high reliability and validity concerning the ADL evaluation after stroke; it consists of $13 \mathrm{~m}$-FIM and 5 cognitive FIM (c-FIM) items [14] [15]. The m-FIM comprises 13 items in 4 subscales: Self-care, Sphincter control, Transfers,

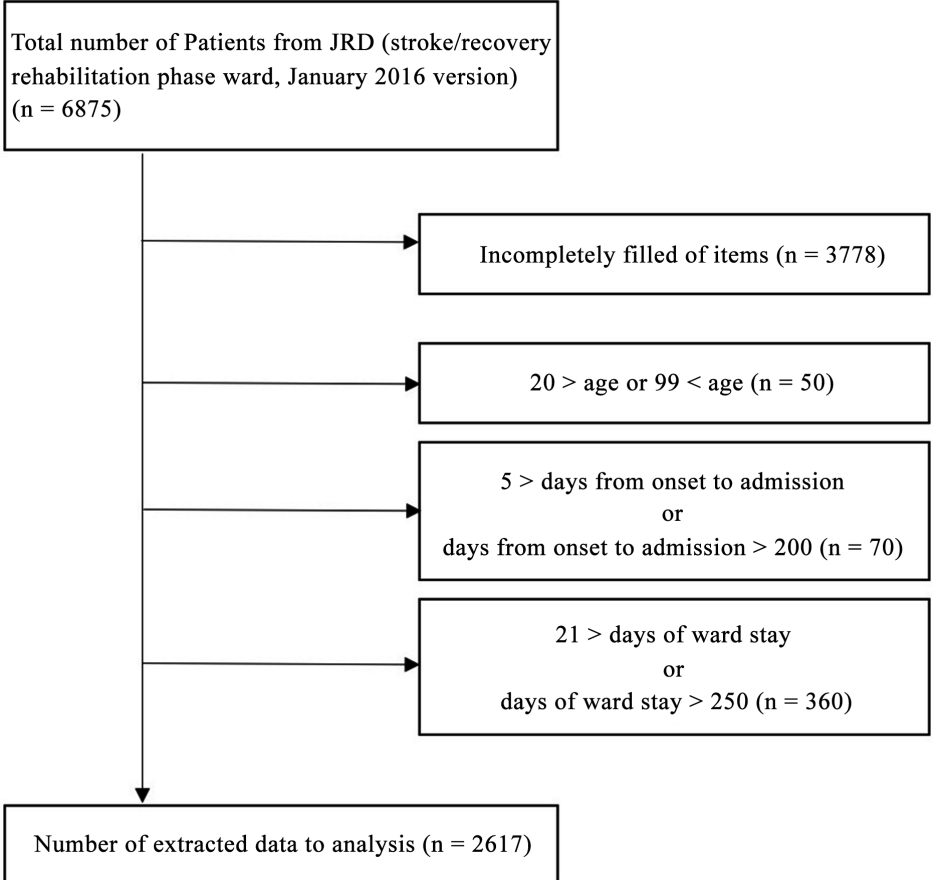

Figure 1. Flowchart of the data sampling with reasons for exclusion. JRD, Japanese rehabilitation database. 
and Locomotion. Self-care includes the activities of eating, grooming, bathing, dressing (upper body and lower body, Toileting), Sphincter control (Bladder and Bowel management), Transfers (Transferring to bed, chair, wheelchair/toilet/, and tub/shower), and Locomotion (Walk or Wheelchair) and Stairs. All items were scored using a 7-point ordinal scale: level 1, Total Assist; level 2, Maximal Assist; level 3, Moderate Assist; level 4, Minimal Assist; level 5, Supervision; level 6, Modified Independence; and level 7, Complete Independence. Caregiving ability was set using a 5-point ordinal scale: level 1, almost none; level 2, between level 1 to 3 ; level 3 , equivalent to be one person who constantly is available to care; level 4, between level 3 to 5; level 5, equivalent to be more than two persons who are constantly available to care. In this study, the destination after leaving the hospital was categorized as follows: home discharge, non-home discharge like nursing facilities etc. The stroke type does not generally influence the prognosis [16] [17]. Therefore, this information was excluded from this study. Sex, the duration from the onset to hospital admission, the length of ward stay, the cognitive FIM score, and caregiving ability were set as the general items.

\subsection{Statical Analysis}

First, all data were modified as mean-centered values to avoid multicollinearity among items. The mean-centering value was calculated by subtracting the mean from the raw value. Second, correlation analyses were performed, and the evaluation of locomotion interaction was subsequently performed using hierarchical multiple regression. Hierarchical regression is a way to show if targeted variables show a statistically significant amount of variance in a dependent variable after accounting for all other variables. In this way, several regression models are built by adding variables to a previous model at each step. This method allows for the examination of the contribution above and beyond the first group of independent variables. In this study, the RS and c-FIM scores were set as the control variables. This analysis used home discharge as the dependent variable in a prediction formula (model 1) using two items as independent variables (RS, c-FIM) to evaluate the correlation of the control variables. Model 2 added two items as independent variables (caregiving ability and self-care). An interaction was considered to occur when the effect of an independent variable on a dependent variable varied across the levels of a moderating variable. Individual self-care score of $\mathrm{m}$-FIM at discharge was set as the moderating variable in this study [17] [18]. Consequently, a prediction formula, namely "model 3," added a mean-centering value to model 2. Finally, a simple slope analysis was performed as a subtest to assess locomotion interaction following the method by Cohen \& Cohen [18] and the Johnson-Neyman technique (JN technique) [19]. In the concept of interaction, the idea of a two-way effect is essential, as opposed to a causal one-way effect. The simple slope method evaluates whether the influence of the independent variable on the dependent variable is statistically significant when the adjustment variable takes a specific value, and has been reported as an effective procedure for investigating the significance of the interaction. That is, fixed val- 
ues of the moderator are chosen, and the significance of X's effect is investigated at those points with a hypothesis test or by constructing a confidence interval. However, when the moderator value is continuous, a better approach is the JN technique. Rather than testing for significance at fixed values of the moderator, the JN technique solves for the values of the moderator for which the effect of $\mathrm{X}$ on $\mathrm{Y}$ becomes or ceases to be significant. Moreover, it can express, as a function of the moderator, the lower and upper bounds for the confidence bands estimating the effect of $\mathrm{X}$ on $\mathrm{Y}$. A graph of the confidence bands makes it easy to see for which values of the moderator the effect of the focal predictor on the response is significant. The statistical significance level was set at $\mathrm{p}<0.05$. Statistical analyses were conducted using IBM SPSS version 20.0 (IBM Corp., Armonk, NY, USA), and the online software created by Preacher, Curran, \& Bauer was used for simple slope analysis.

\section{Results}

In total, the data of 6875 stroke patients were registered on JRD. Of these, the 4258 patients that failed to meet the inclusion criteria were excluded and the 2617 that met the criteria were ultimately included in this study. Among all of participants in this study, $1092(41.7 \%)$ were female and the median age was 69.28 years. RS, m-FIM and c-FIM scores at discharge were 2.60, 68.87 and 26.90, respectively. The baseline characteristics of the subjects in each group (home discharge group: FIM level of self-care showed more than supervision excepting climbing stairs; non-home discharge: there was moderate assistance for the main FIM level and maximal assistance for climbing stairs) are shown in Table 1 . The home discharge rate was $84.5 \%$ and multiple comparisons of all items were statistically significant. Table 2 shows the details of the correlation between all items and individual self-care correlated with home discharge. A hierarchical multiple regression showed the following interaction: grooming, bathing, gait, and climbing stairs (Table 3). Figure 2 illustrates the interaction, that is, the relationship between the independent and dependent values under moderating value variation (means $\pm 1 \mathrm{SD}$ ). The simple slope analysis was significant for four self-care items (grooming, bathing, gait, and climbing stairs). However, it has been reported that a subtest of interaction was limited in the case of a continuous variable. For this reason, Figure 3 presents a region of significance and $95 \%$ confidence band of simple slope in each group. The value of the self-care range was relevant to the lower or over the upper bound region in all items and it was significant.

\section{Discussion}

This study investigated the interaction between self-care and caregiving ability in stroke patients based on the m-FIM score at discharge using multi-facility data (JRD). The main finding suggested that self-care and caregiving ability in two groups, which were classified based on the discharge destination, had an interaction. The aspects of self-care that influenced home discharge were the following: 
Table 1. Descriptive characteristics of the subject at discharge.

\begin{tabular}{|c|c|c|c|}
\hline & $\begin{array}{c}\text { Overall } \\
(\mathrm{n}=2617)\end{array}$ & $\begin{array}{l}\text { Non-home discharge } \\
\qquad(\mathrm{n}=405)\end{array}$ & $\begin{array}{l}\text { Home discharge } \\
\qquad(\mathrm{n}=2212)\end{array}$ \\
\hline Age & $69.28 \pm 12.89$ & $76.22 \pm 9.43$ & $68.01 \pm 13.03^{* *}$ \\
\hline Female, \%, (n) & $41.72(1092)$ & $47.40(192)$ & $40.69(900)$ \\
\hline Days form onset to admission & $34.80 \pm 14.67$ & $37.75 \pm 14.33$ & $34.26 \pm 14.68^{* *}$ \\
\hline Days of ward stay & $101.79 \pm 44.44$ & $118.40 \pm 40.97$ & $98.75 \pm 44.38^{\star \star}$ \\
\hline Caregiving ability & $2.27 \pm 0.96$ & $1.82 \pm 0.94$ & $2.36 \pm 0.94^{* *}$ \\
\hline Rankin scale & $2.60 \pm 1.18$ & $3.54 \pm 1.03$ & $2.42 \pm 1.13^{* *}$ \\
\hline m-FIM & $68.87 \pm 19.00$ & $49.52 \pm 21.23$ & $73.59 \pm 16.89^{* *}$ \\
\hline c-FIM & $26.90 \pm 7.40$ & $20.68 \pm 7.47$ & $28.03 \pm 6.79^{* *}$ \\
\hline Eating & $6.21 \pm 1.20$ & $5.36 \pm 1.50$ & $6.37 \pm 1.06^{* *}$ \\
\hline Grooming & $5.84 \pm 1.59$ & $4.42 \pm 1.89$ & $6.10 \pm 1.38^{\star *}$ \\
\hline Bathing & $4.68 \pm 1.94$ & $2.93 \pm 1.76$ & $5.00 \pm 1.79^{* *}$ \\
\hline Dressing upper body & $5.64 \pm 1.83$ & $3.85 \pm 2.06$ & $5.97 \pm 1.58^{\star *}$ \\
\hline Dressing lower body & $5.47 \pm 1.99$ & $3.48 \pm 2.16$ & $5.83 \pm 1.73^{\star *}$ \\
\hline Toileting & $5.62 \pm 1.80$ & $3.87 \pm 2.16$ & $5.94 \pm 1.52^{\star *}$ \\
\hline Bladder management & $5.69 \pm 1.96$ & $3.86 \pm 2.27$ & $6.02 \pm 1.70^{* *}$ \\
\hline Bowel management & $5.71 \pm 1.73$ & $4.15 \pm 2.16$ & $6.00 \pm 1.47^{* *}$ \\
\hline Gait/Wheel chair & $5.05 \pm 2.02$ & $3.70 \pm 2.14$ & $5.30 \pm 1.90^{\star *}$ \\
\hline Climbing stars & $3.90 \pm 2.26$ & $2.15 \pm 1.81$ & $4.21 \pm 2.19^{* *}$ \\
\hline
\end{tabular}

m-FIM, Functional Independent Measure; motor c-FIM, cognitive Functional Independent Measure; mean \pm SD, median \pm standard deviation. ${ }^{* *}: \mathrm{p}<0.01$.

Table 2. Correlation between all items at discharge after mean-centering.

\begin{tabular}{|c|c|c|c|c|c|c|c|c|c|c|c|c|c|c|c|c|c|}
\hline & 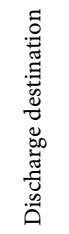 & $\stackrel{0}{4}$ & 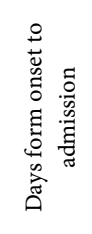 & 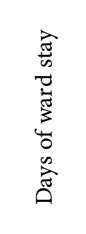 & 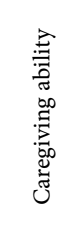 & 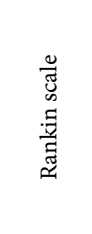 & 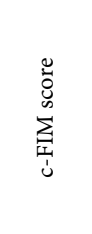 & 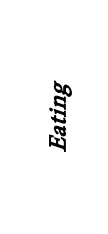 & 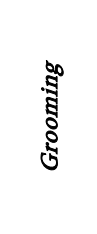 & 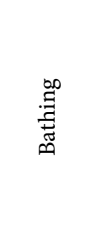 & 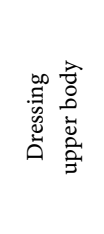 & 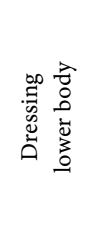 & 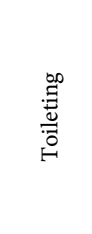 & 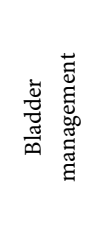 & 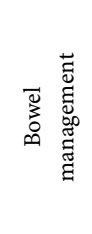 & 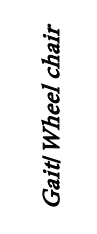 & 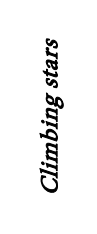 \\
\hline $\begin{array}{l}\text { Discharge } \\
\text { destination }\end{array}$ & 1.00 & $-0.23^{* *}$ & $-0.09^{* *}$ & $-0.16^{* *}$ & $0.20^{* *}$ & $-0.34^{* *}$ & $0.36^{* *}$ & $0.30^{* *}$ & $0.38^{* *}$ & $0.39^{* *}$ & $0.42^{\star *}$ & $0.43^{* *}$ & $0.42^{* *}$ & $0.40^{* *}$ & $0.39^{* *}$ & $0.29^{* *}$ & $0.33^{* *}$ \\
\hline Age & & 1.00 & -0.03 & $-0.04^{*}$ & 0.03 & $0.24^{* *}$ & $-0.29 * *$ & $-0.23^{* *}$ & $-0.27^{* *}$ & $-0.29 * *$ & $-0.26^{\star *}$ & $-0.27^{* *}$ & $-0.27^{* *}$ & $-0.31^{* *}$ & $-0.29 * *$ & $-0.25^{* *}$ & $-0.28^{* *}$ \\
\hline $\begin{array}{l}\text { Days form onset } \\
\text { to admission }\end{array}$ & & & 1.00 & $0.17^{* *}$ & $0.05^{*}$ & $0.18^{* *}$ & $-0.22^{* *}$ & $-0.16^{* *}$ & $-0.18^{* *}$ & $-0.17^{* *}$ & $-0.18^{\star *}$ & $-0.18^{\star *}$ & $-0.19^{* *}$ & $-0.17^{* *}$ & $-0.17^{* *}$ & $-0.12^{* *}$ & $-0.17^{* *}$ \\
\hline Days of ward stay & & & & 1.00 & 0.02 & $0.41^{\star *}$ & $-0.19^{* *}$ & $-0.25^{* *}$ & $-0.27^{* *}$ & $-0.39^{* *}$ & $-0.30^{\star *}$ & $-0.33^{* *}$ & $-0.32^{* *}$ & $-0.25^{* *}$ & $-0.24^{* *}$ & $-0.22^{* *}$ & $-0.37^{* *}$ \\
\hline Caregiving ability & & & & & 1.00 & $0.07^{* *}$ & $-0.09 * *$ & $-0.09 * *$ & $-0.09 * *$ & $-0.08^{* *}$ & $-0.075^{* *}$ & $-0.08^{* *}$ & $-0.09^{* *}$ & $-0.10^{* *}$ & $-0.10^{* *}$ & $-0.08^{* *}$ & $-0.07 * *$ \\
\hline Rankin scale & & & & & & 1.00 & $-0.51^{* *}$ & $-0.49^{* *}$ & $-0.59^{* *}$ & $-0.70^{* *}$ & $-0.64^{* *}$ & $-0.66^{* *}$ & $-0.64^{* *}$ & $-0.56^{* *}$ & $-0.55^{* *}$ & $-0.52^{* *}$ & $-0.63^{* *}$ \\
\hline c-FIM & & & & & & & 1.00 & $0.58^{* *}$ & $0.69^{* *}$ & $0.62^{* *}$ & $0.63^{* *}$ & $0.63^{* *}$ & $0.62^{* *}$ & $0.65^{* *}$ & $0.65^{* *}$ & $0.44^{* *}$ & $0.45^{* *}$ \\
\hline Eating & & & & & & & & 1.00 & $0.75^{* *}$ & $0.64^{* *}$ & $0.67^{* *}$ & $0.67^{* *}$ & $0.68^{* *}$ & $0.64^{* *}$ & $0.64^{* *}$ & $0.50^{* *}$ & $0.49^{* *}$ \\
\hline Grooming & & & & & & & & & 1.00 & $0.76^{* *}$ & $0.82^{* *}$ & $0.81^{* *}$ & $0.80^{* *}$ & $0.75^{* *}$ & $0.73^{* *}$ & $0.58^{* *}$ & $0.56^{* *}$ \\
\hline Bathing & & & & & & & & & & 1.00 & $0.80^{* *}$ & $0.81^{* *}$ & $0.79^{* *}$ & $0.70^{* *}$ & $0.67^{* *}$ & $0.57^{* *}$ & $0.70^{* *}$ \\
\hline Dressing upper body & & & & & & & & & & & 1.00 & $0.96^{* *}$ & $0.85^{* *}$ & $0.75^{* *}$ & $0.73^{* *}$ & $0.59^{* *}$ & $0.61^{* *}$ \\
\hline Dressing lower body & & & & & & & & & & & & 1.00 & $0.87^{* *}$ & $0.76^{* *}$ & $0.73^{* *}$ & $0.60^{* *}$ & $0.63^{* *}$ \\
\hline Toileting & & & & & & & & & & & & & 1.00 & $0.79 * *$ & $0.77^{* *}$ & $0.62^{* *}$ & $0.62^{* *}$ \\
\hline Bladder anagement & & & & & & & & & & & & & & 1.00 & $0.85^{* *}$ & $0.57^{* *}$ & $0.57^{* *}$ \\
\hline Bowel management & & & & & & & & & & & & & & & 1.00 & $0.56^{* *}$ & $0.53^{* *}$ \\
\hline Gait/Wheel chair & & & & & & & & & & & & & & & & 1.00 & $0.51^{* *}$ \\
\hline Climbing stars & & & & & & & & & & & & & & & & & 1.00 \\
\hline
\end{tabular}

c-FIM, cognitive Functional Independence Measure; Bold and Italic word, item that had an interaction in this study. ${ }^{*} ; \mathrm{p}<0.05,{ }^{* *} ; \mathrm{p}<0.01$. 


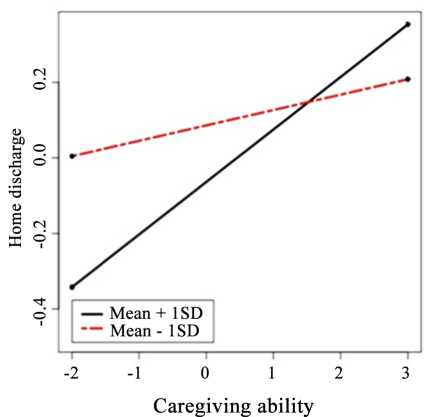

Mean - 1SD: $y=0.04 \times$ caregiving ability $+0.09(\mathrm{p}<0.001)$ Mean +1 SD: $y=0.14 \times$ caregiving ability $-0.06(\mathrm{p}<0.001)$

(a)

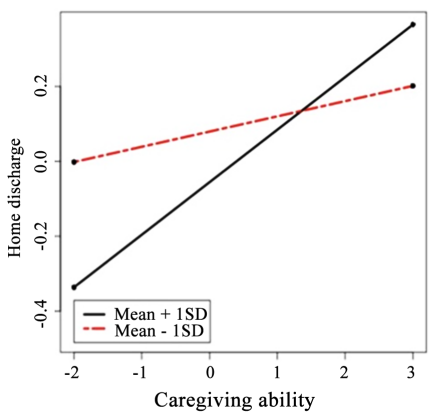

Mean - 1SD: $y=0.04 \times$ caregiving ability $+0.08(p<0.001)$ Mean + 1SD: $y=0.14 \times$ caregiving ability $-0.06(p<0.001)$

(b)

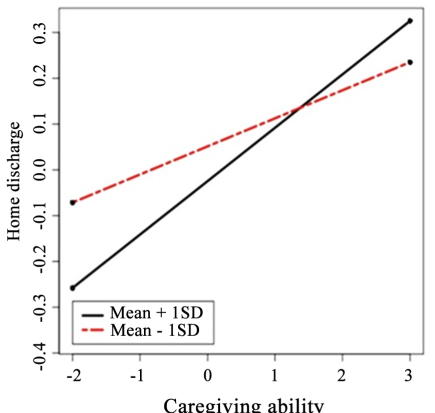

Mean - 1SD: $y=0.06 \times$ caregiving ability $+0.05(p<0.001)$ Mean + 1SD: $y=0.12 \times$ caregiving ability $-0.02(\mathrm{p}<0.001)$ (c)

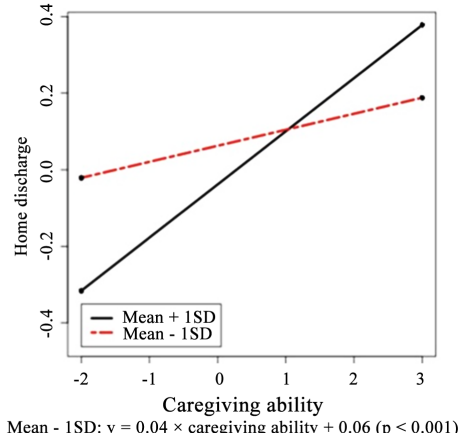

$y=0.04 \times$ caregiving ability $+0.06(p<0.0$ (d)

Figure 2. A result of simple slope analysis in each self-care after mean-centering. (a) grooming; (b) Bathing; (c) Gait/Wheel chair; (d) Climbing stairs.

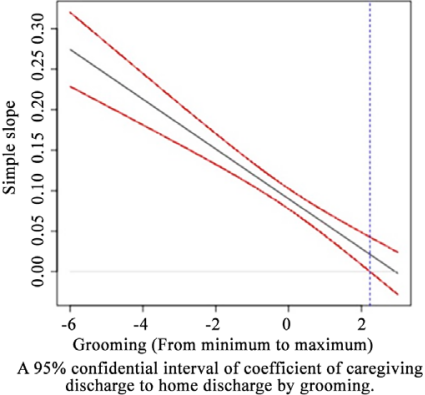

(a)

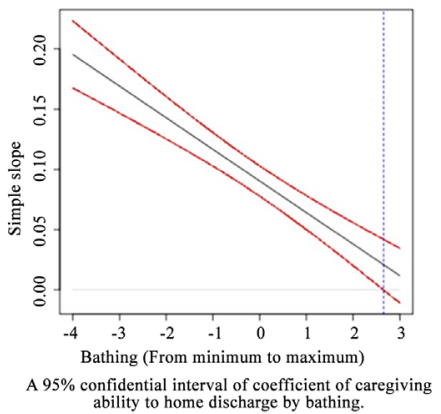

(b)

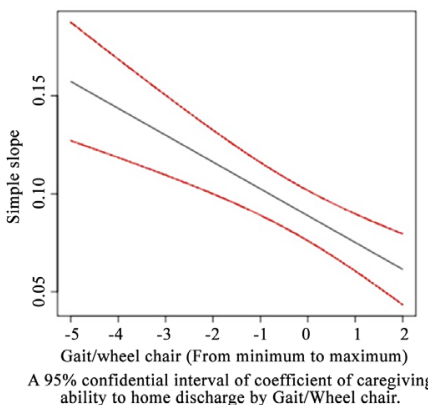

(c)

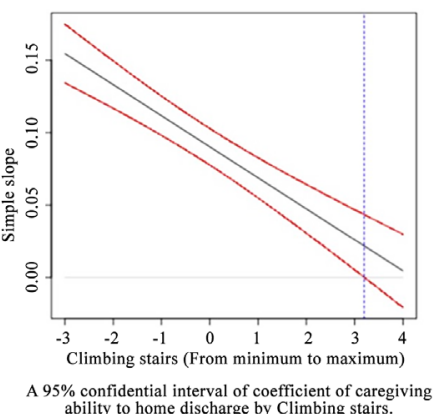

(d)

Figure 3. A region of significance and $95 \%$ confidence band of Simple slope after mean-centering. Center line with grey color; Prediction coefficient of simple slope, Up and down curve line with red color; 95\% confidence interval of prediction coefficient, Vertical line with blue; lower or upper bound of region. (a) grooming; (b) Bathing; (c) Gait/Wheel chair; (d) Climbing stairs. 
Table 3. The results of hierarchical multiple regression analysis for individual self-care.

\begin{tabular}{|c|c|c|c|c|c|c|c|c|c|}
\hline \multicolumn{10}{|l|}{ 1. Grooming } \\
\hline \multirow{2}{*}{ Variable } & \multicolumn{3}{|c|}{ Step 1} & \multicolumn{3}{|c|}{ Step 2} & \multicolumn{3}{|c|}{ Step 3} \\
\hline & $\mathrm{b}$ & & bSE & $\mathrm{b}$ & & bSE & $\mathrm{b}$ & & bSE \\
\hline \multicolumn{10}{|l|}{ Step 1} \\
\hline RS & -0.065 & $* *$ & 0.006 & -0.049 & $* *$ & 0.007 & 0.012 & $* *$ & 0.004 \\
\hline c-FIM & 0.012 & $* *$ & 0.001 & 0.008 & $* *$ & 0.001 & 0.014 & $* *$ & 0.004 \\
\hline \multicolumn{10}{|l|}{ Step 2} \\
\hline Caregiving ability & & & & 0.092 & $* *$ & 0.006 & 0.505 & $* *$ & 0.019 \\
\hline Grooming & & & & 0.045 & $* *$ & 0.006 & 0.177 & $* *$ & 0.018 \\
\hline \multicolumn{10}{|l|}{ Step 3} \\
\hline \multicolumn{10}{|l|}{ Caregiving ability } \\
\hline \multicolumn{10}{|l|}{$\times$ Grooming } \\
\hline$\Delta \mathrm{R}^{2}$ & & & & 0.075 & $* *$ & & 0.019 & $* *$ & \\
\hline Adj $R^{2}$ & & & & 0.237 & & & 0.255 & & \\
\hline \multicolumn{10}{|l|}{ 2. Bathing } \\
\hline \multirow{2}{*}{ Variable } & \multicolumn{3}{|c|}{ Step 1} & \multicolumn{3}{|c|}{ Step 2} & \multicolumn{3}{|c|}{ Step 3} \\
\hline & $\mathrm{b}$ & & bSE & $\mathrm{b}$ & & bSE & $\mathrm{b}$ & & bSE \\
\hline \multicolumn{10}{|l|}{ Step 1} \\
\hline RS & -0.065 & $* *$ & 0.006 & -0.036 & $* *$ & 0.007 & -0.033 & $* *$ & 0.007 \\
\hline c-FIM & 0.012 & $* *$ & 0.001 & 0.010 & $* *$ & 0.001 & 0.010 & $* *$ & 0.001 \\
\hline \multicolumn{10}{|l|}{ Step 2} \\
\hline Caregiving ability & & & & 0.091 & $* *$ & 0.006 & 0.090 & $* *$ & 0.006 \\
\hline Bathing & & & & 0.038 & $* *$ & 0.005 & 0.036 & $* *$ & 0.005 \\
\hline \multicolumn{10}{|l|}{ Step 3} \\
\hline \multicolumn{10}{|l|}{ Caregiving ability } \\
\hline \multicolumn{10}{|l|}{$\times$ Bathing } \\
\hline$\Delta \mathrm{R}^{2}$ & & & & 0.074 & $* *$ & & 0.019 & $* *$ & \\
\hline Adj $R^{2}$ & & & & 0.236 & & & 0.255 & & \\
\hline
\end{tabular}

\begin{tabular}{|c|c|c|c|c|c|c|c|c|c|}
\hline \multicolumn{10}{|l|}{ 3. Gait/Wheel chair } \\
\hline \multirow{2}{*}{ Variable } & \multicolumn{3}{|c|}{ Step 1} & \multicolumn{3}{|c|}{ Step 2} & \multicolumn{3}{|c|}{ Step 3} \\
\hline & $\mathrm{b}$ & & bSE & $\mathrm{b}$ & & bSE & $\mathrm{b}$ & & bSE \\
\hline \multicolumn{10}{|l|}{ Step 1} \\
\hline RS & -0.065 & $* *$ & 0.006 & -0.055 & $* *$ & 0.007 & -0.052 & $* *$ & 0.007 \\
\hline c-FIM & 0.012 & $* *$ & 0.001 & 0.012 & $* *$ & 0.001 & 0.012 & $* *$ & 0.001 \\
\hline \multicolumn{10}{|l|}{ Step 2} \\
\hline Caregiving ability & & & & 0.092 & $* *$ & 0.007 & 0.089 & $* *$ & 0.007 \\
\hline Gait/Wheel chair & & & & 0.019 & ** & 0.004 & 0.019 & $* *$ & 0.004 \\
\hline
\end{tabular}




\section{Continued}

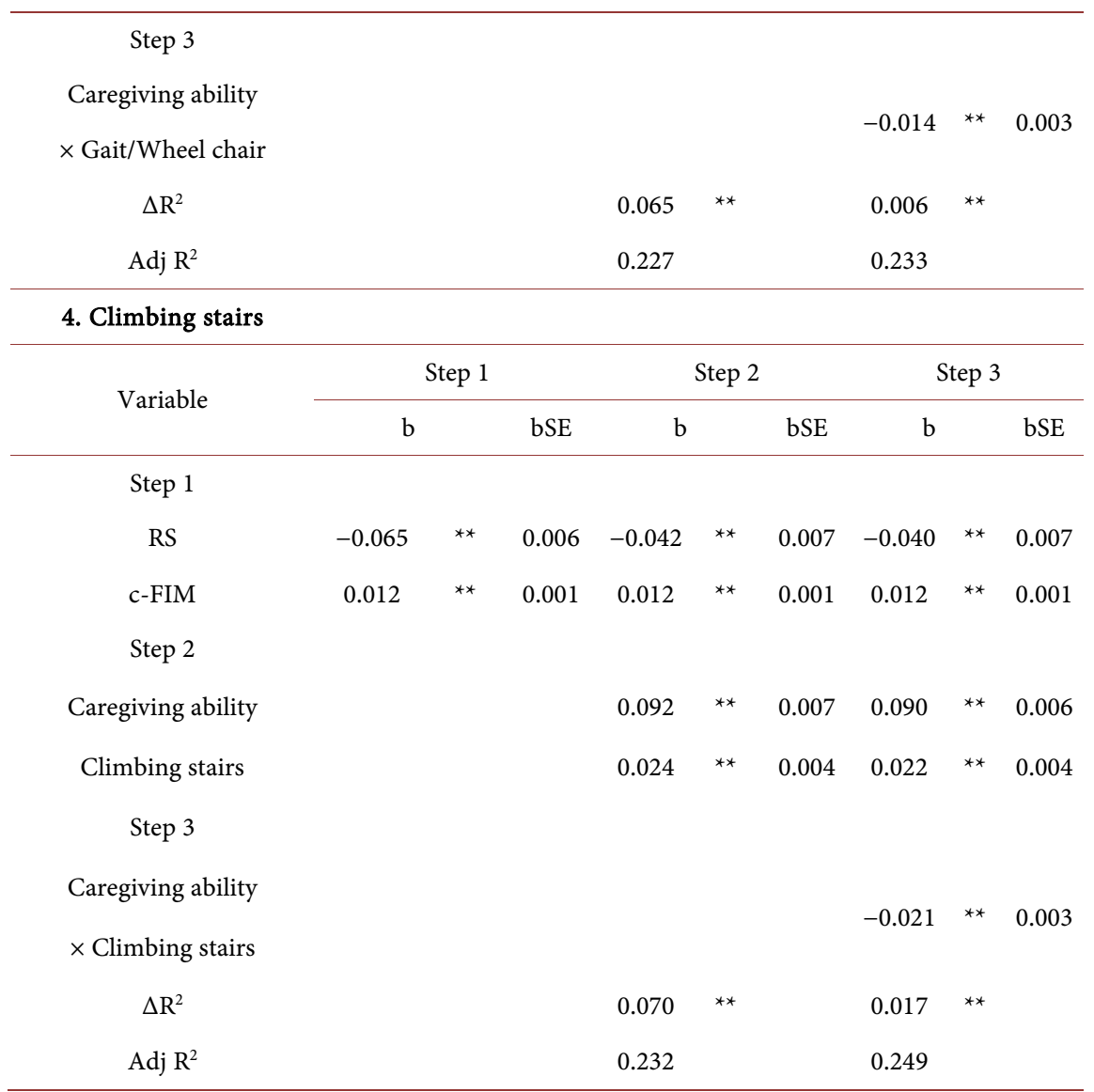

RS, Rankin Scale; c-FIM, cognitive Functional Independent Measure. ${ }^{* *}$; $\mathrm{p}<0.01$.

grooming, bathing, gait, and climbing stairs. Stroke is one of the diseases that require daily living care. Caregiving ability is composed of physical, emotional, economic, and social factors. In addition, it is reported that improving ADL level influences the care burden and discharge destination. FIM is widely used to predict home discharge, and a previous study reported that the average scores of $\mathrm{m}$-FIM and c-FIM for stroke patients who could be discharged to their homes regardless of whether they are living alone or with their family was 66.2 and 25.7, respectively [20]. Moreover, the Receiver Operating Characteristic analysis showed that the cutoff points of m-FIM and c-FIM for stroke patients who returned home to live alone were 78 and 28, respectively [9]. Both average scores in this study were similar to the previous studies. Additionally, the home discharge rate was like those of previous studies as well. It is generally seen that basic actions in daily living in the order of difficulty are rolling over in bed, sitting up, standing up, and walking. However, the order of post-stroke recovery is not always similar and differs according to the patients' developmental stage; in addition, it can be influenced by age and physical paralysis.

Furthermore, it has been reported that grooming, bathing, gait, and climbing stairs in ADL disturbance have a higher burden of care than other self-care such as eating [21] [22] [23]. Moreover, only 52\% of caregivers were confident in 
caring for stroke patients, despite their knowledge about stroke-related nursing care [22] [24]. For these reasons, it is assumed that the caregiver's burden depends on the independence level of self-care and influence on home discharge.

These aspects of self-care are necessary for stroke patients living in their homes. However, the independence level of the above-mentioned self-care activities is difficult to improve, except for grooming [25] [26].

The role and development of grooming behavior in daily living are necessary for forming social relationships in the community. Regarding oral care, an aspect of grooming, it was reported that $83.9 \%$ of stroke patients had difficulty brushing their teeth and were dependent on caregivers to maintain oral health [27]. Behavior involves being conscious of others; for instance, grooming consumes approximately $20 \%$ of daily time toward maintaining social relationships [28] [29] [30]. Therefore, it is possible that grooming had a high daily frequency, despite taking less time than other activities. This may result in having to take care of stroke patients anywhere and anytime, therefore increasing the family caregivers' burden. This indicates that the caregivers' burden depends on the independence level of grooming. Consequently, it is assumed that grooming presented an interaction, although its independence level is easier to improve than for bathing, gait, and climbing stairs and it is the most common basic activity in daily life.

Bathing had a similarly strong interaction with other self-care areas in this study. Bathing is one of the most difficult actions and requires the caregiver to be strong enough to carry the physical burden even while using a shower chair instead of a bathtub [27] [31]. It was assumed that the bathing process, which includes related activities like standing, grooming, and transfer has an influence on the burden as well [31] [32].

Stroke patients experience extreme difficulty with even the slightest difference level. The environmental situation at home, such as steps within the building, is considered to be different from the safety-adjusted environment of the hospital. Therefore, this may cause falls in stroke patients and may be one of the obstacles in improving their independence to self-care activities. Gait and ADL disturbances were reported as factors causing falls in home-dwelling stroke patients. Additionally, a relationship between gait speed, balance at hospitalization, and falling in home-dwelling stroke patients was reported. Furthermore, on discharge from hospital, both scores of capability and performance in ADL were different, and the agreement rate was lower than in other areas [25]. Moreover, gait ability is easily influenced not only by physical factors but also external factors such as home environment [33] [34]. Additionally, stroke patients have a high risk of falling at their homes and reported fallers might return to the hospital [35] [36] [37]. For this reason, caring for stroke patients at home is assumed to require a high level of caregiving ability to avoid falling.

Gait, which is a major disturbing factor for patients discharged to their homes post-stroke, is defined as moving from one place to another and requires a highly applied balance control. Moreover, gait is reported to be an important factor 
for daily living. It is a useful indicator of post-stroke prognosis and can influence discharge destination. Therefore, it should focus on an activity like this. Hence, maintaining gait requires bearing high caregiving burden through physical help to avoid falls in stroke patients.

As with gait, climbing stairs was reported as a major cause of the high risk of falling in home-dwelling stroke patients [31] [38] and had the widest gaps between capacity and performance of ADL at discharge [25]. Furthermore, the applied balance ability to stand on one leg is needed while climbing stairs. Such an advanced balance ability is one of the most difficult activities and is hard to perform at an independent level for stroke patients. Therefore, this situation strains caregivers as it takes great effort to ensure that the stroke patient does not fall even due to a slight step. The knee joint was reported to be highly loaded when climbing the stairs, with an average peak resultant force, in percent of body weight, three times higher compared with gait [39], indicating that caregiving burden is greater while climbing stairs. Standing with one foot when climbing the stairs, it is required even when stroke patients get over different level in the home, which also applies considerable pressure on the knee. Thus, it requires more direct caregiving as well as advanced techniques compared to other self-care activities. Moreover, falling and fall-related injuries are reported to be serious problems for home-dwelling stroke patients [40]. For these reasons, falling is a negative factor and is assumed to lead to negative feelings such as perceived rejection by family members about home discharge. Therefore, it is assumed that the ability to independently climb stairs influences discharge.

It has been reported that climbing stairs was not more highly correlated to home discharge when compared to other types of self-care, including gait, which was considered one type of extraordinary activity in previous studies [2] [25] [39]. Therefore, interventions for home discharge for post-stroke patients have mainly tended to target gait. However, climbing stairs was shown to have an interaction with home discharge in this study. Climbing stairs is an activity that requires more applied balance than gait or self-care, and it has been reported that lower limb function that is used in climbing stairs influences these abilities [2] [36] [39]. For this reason, working toward more difficult activities could increase abilities to engage in self-care activities, and a focus on improving climbing stairs could be effective in preparing for home discharge and should be positively considered for stroke patients.

A limitation to this study is that RS and c-FIM scores were set as the control variables in all groups without considering m-FIM upon discharge; therefore, the characteristics of each group were not considered in setting the control variables for that particular group. Hence, future research should investigate the interaction between self-care and caregiving ability using control variables that reflect the group characteristics or after hierarchy with m-FIM upon discharge.

In conclusion, this study investigated the interaction of self-care and caregiving ability in two groups that were classified based on the discharge destination. Four areas, namely, grooming, bathing, gait, and climbing stairs, had an interac- 
tion based on the m-FIM at discharge according to the JRD; these self-care practices exerted a great burden or influence on caregivers and home discharge. Therefore, this study's findings suggest the need for an intervention that focuses on improving ADL for stroke patients that live at their home, especially climbing stairs. Future research should be conducted in hierarchy with the number of caregivers or m-FIM score upon discharge and should further test and discuss the influencing factors.

\section{Acknowledgements}

This study used the Japan Rehabilitation Database managed by the Japan Association of Rehabilitation Database, to whom we express our gratitude. The contents and conclusions of this study are not the opinions of the Association but exclusively those of the authors.

\section{Funding}

This research received no specific grant from any funding agency in the public, commercial, or not-for-profit sectors.

\section{Conflicts of Interest}

The author declares no conflicts of interest regarding the publication of this paper.

\section{References}

[1] Matsuyama, A. (2018) Factors Associated with the Walking Ability of Hemiplegic Stroke Patients. Open Journal of Nursing, 8, 14-25. https://doi.org/10.4236/ojn.2018.81002

[2] Eng, J.J. and Tang, P.F. (2007) Gait Training Strategies to Optimize Walking Ability in People with Stroke: A Synthesis of the Evidence. Expert Review of Neurotherapeutics, 7, 1417-1436. https://doi.org/10.1586/14737175.7.10.1417

[3] Aşiret, G.D. and Kapucu, S. (2013) Burden of Caregivers of Stroke Patients. Turkish Journal of Neurology, 19, 5-10. https://doi.org/10.4274/Tnd.60234

[4] Ugur, H.G. and Erci, B. (2019) The Effect of Home Care for Stroke Patients and Education of Caregivers on the Caregiver Burden and Quality of Life. Acta clinica Croatica, 58, 321-332.

[5] Costa, T.F., Costa, K.N., Fernandes, M.D., Martins, K.P. and Brito, S.D.S. (2015) Quality of Life of Caregivers for Patients of Cerebrovascular Accidents: Association of (Socio-Demographic) Characteristics and Burden. Revista da Escola de Enfermagem da USP, 49, 245-252. https://doi.org/10.1590/S0080-623420150000200009

[6] Sato, A., Fujita, T. and Yamamoto, Y. (2017) Activities of Daily Living Independence Level for Home Discharge in Stroke Patients Based on Number of Caregivers: an Analysis of the Japan Rehabilitation Database. Physical Therapy Research, 20 23-27. https://doi.org/10.1298/ptr.E9914

[7] Sato, K. (2020) Predictive Factors of Home Discharge in Elderly Stroke Patients Hospitalized in a Convalescent Rehabilitation Ward. Japanese Journal of Comprehensive Rehabilitation Science, 11, 43-48. 
[8] Nguyen, T.A., Page, A., Aggarwal, A. and Henke, P. (2007) Social Determinants of Discharge Destination for Patients after Stroke with Low Admission FIM Instrument Scores. Archives of Physical Medicine and Rehabilitation, 88, 740-744. https://doi.org/10.1016/j.apmr.2007.03.011

[9] Miura, H., Shimizu, S., Noma, T., Ichinosawa, Y., Shimose, R., Tsunoda, S., et al. (2018) Post-Stroke Patients' Activities of Daily Living Levels for Discharge to Return Home to Live Alone. The Kitasato Medical Journal, 48, 118-127.

[10] Koyama, T., Sako, Y., Konta, M. and Domen, K. (2011) Poststroke Discharge Destination: Functional Independence and Sociod Emographic Factors in Urban Japan. Journal of Stroke and Cerebrovascular Diseases, 20, 202-207. https://doi.org/10.1016/j.jstrokecerebrovasdis.2009.11.020

[11] Iwai, N. and Aoyagi, Y. (2012) Discharge Index and Prediction for Stroke Patients in the Post-Acute Stage: Evaluation of the Usefulness of Nichijo-Seikatu-KinoHyokahyo. Japanese Journal of Comprehensive Rehabilitation Science, 3, 37-41.

[12] Nguyen, V.Q., PrvuBettger, J., Guerrier, T., Hirsch, M.A., Thomas, J.G., Pugh, T.M., et al. (2015) Factors Associated with Discharge to Home Versus Discharge to Institutional Care after Inpatient Stroke Rehabilitation. Archives of Physical Medicine and Rehabilitation, 96, 1297-1303. https://doi.org/10.1016/j.apmr.2015.03.007

[13] Jaracz, K., Grabowska-Fudala, B., Górna, K. and Kozubski, W. (2014) Consequences of Stroke in the Light of Objective and Subjective Indices: A Review of Recent Literature. Neurologia i Neurochirurgia Polska, 48, 280-286.

https://doi.org/10.1016/j.pjnns.2014.07.004

[14] Ottenbacher, K.J., Hsu, Y., Granger, C.V. and Fiedler, R.C. (1996) The Reliability of the Functional Independence Measure: A Quantitative Review. Archives of Physical Medicine and Rehabilitation, 77, 1226-1232.

https://doi.org/10.1016/S0003-9993(96)90184-7

[15] Chen, H.F., Wu, C.Y., Lin, K.C., Chen, C.L., Huang, P.C., et al. (2013) Rasch Validation of a Combined Measure of Basic and Extended Daily Life Functioning after Stroke. Neurorehabil Neural Repair, 27, 125-132.

https://doi.org/10.1177/1545968312457828

[16] Lopes, P.G., Lopes, J.A., Brito, C.M., Alfieri, F.M. and Battistella, R.L. (2015) Relationships of Balance, Gait Performance, and Functional Outcome in Chronic Stroke Patients: A Comparison of Left and Right Lesions. Biomedical Research, 2015, Article ID: 716042. https://doi.org/10.1155/2015/716042

[17] Jørgensen, H.S., Nakayama, H., Raaschou, H.O. and Olsen, T.S. (1995) Intracerebral Hemorrhage Versus Infarction: Stroke Severity, Risk Factors, and Prognosis. Annals of Neurology, 38, 45-50. https://doi.org/10.1002/ana.410380110

[18] Brankovic, M., Kardys, I., Steyerberg, E.W., Lemeshow, S., Markovic, M., et al. (2019) Understanding of Interaction (Subgroup) Analysis in Clinical Trials. European Journal of Clinical Investigation, 49, e13145. https://doi.org/10.1111/eci.13145

[19] Andersson, U., Cuervo-Cazurra, A. and Nielsen, B.B. (2014) Explaining Interaction Effects within and across Levels of Analysis. Journal of International Business Studies, 45, 1063-1071. https://doi.org/10.1057/jibs.2014.50

[20] Mutai, H., Furukawa, T., Araki, K., Misawa, K. and Hanihara, T. (2012) Factors Associated with Functional Recovery and Home Discharge in Stroke Patients Admitted to a Convalescent Rehabilitation Ward. Geriatrics \& Gerontology International, 12, 215-222. https://doi.org/10.1111/j.1447-0594.2011.00747.x

[21] Bhattacharjee, M., Vairale, J., Gawali, K. and Dalal, P. M. (2012) Factors Affecting Burden on Caregivers of Stroke Survivors: Population-Based Study in Mumbai (In- 
dia). Annals of Indian Academy of Neurology, 15, 113-119. https://doi.org/10.4103/0972-2327.94994

[22] Menon, B., Salini, P., Habeeba, K., Conjeevaram, J. and Munisusmitha, K. (2017) Female Caregivers and Stroke Severity Determines Caregiver Stress in Stroke Patients. Annals of Indian Academy of Neurology, 20, 418-424.

https://doi.org/10.4103/aian.AIAN_203_17

[23] Jaracz, K., Grabowska-Fudala, B., Górna, K. and Kozubski, W. (2014) Caregiving Burden and Its Determinants in Polish Caregivers of Stroke Survivors. Archives of Medical Science, 10, 941-950. https://doi.org/10.5114/aoms.2014.46214

[24] Tsai, Y.H., Lou, M.F., Feng, T.H., Chu, T.L., Chen, Y.J., et al. (2018) Mediating Effects of Burden on Quality of Life for Caregivers of First-Time Stroke Patients Discharged from the Hospital within One Year. BMC Neurology, 18, Article No. 50. https://doi.org/10.1186/s12883-018-1057-9

[25] Iwai, N., Aoyagi, Y., Tokuhisa, K., Yamamoto, J. and Shimada, T. (2011) The Gaps between Capability ADL and Performance ADL of Stroke Patients in a Convalescent Rehabilitation Ward-Based on the Functional Independence Measure. The Journal of Physical Therapy Science, 23, 333-338. https://doi.org/10.1589/jpts.23.333

[26] Koyama, T., Matsumoto, K., Okuno, T. and Domen, K. (2006) Relationships between Independence Level of Single Motor-FIM Items and FIM-Motor Scores in Patients with Hemiplegia after Stroke: An Ordinal Logistic Modelling Study. Journal of Rehabilitation Medicine, 38, 280-286. https://doi.org/10.1080/16501970600731420

[27] Morone, G., Paolucci, S. and Iosa, M. (2015) In What Daily Activities Do Patients Achieve Independence after Stroke? Journal of Stroke and Cerebrovascular Diseases, 24, 1931-1937. https://doi.org/10.1016/j.jstrokecerebrovasdis.2015.05.006

[28] Roberts, S.G.B. and Dunbar, R.I.M. (2011) Communication in Social Networks: Effects of Kinship, Network Size, and Emotional Closeness. Personal Relationships, 18, 439-452. https://doi.org/10.1111/j.1475-6811.2010.01310.x

[29] Masanori, T. (2018) Two Types of Social Grooming Methods Depending on the Trade-Off between the Number and Strength of Social Relationships. Royal Society Open Science, 5, Article No. 5180148. https://doi.org/10.1098/rsos.180148

[30] Kwok, C., McIntyre, A., Janzen, S., Mays, R. and Teasell, R. (2015) Oral Care Post Stroke: A Scoping Review. Journal of Oral Rehabilitation, 42, 65-74. https://doi.org/10.1111/joor.12229

[31] Wolfe, C.D. (2000) The Impact of Stroke. British Medical Bulletin, 56, 275-286. https://doi.org/10.1258/0007142001903120

[32] Edwards, N., Dulai, J. and Rahman, A. (2019) A Scoping Review of Epidemiological, Ergonomic, and Longitudinal Cohort Studies Examining the Links between Stair and Bathroom Falls and the Built Environment. International Journal of Environmental Research and Public Health, 16, 1598.

https://doi.org/10.3390/ijerph16091598

[33] Goto, Y., Otaka, Y., Suzuki, K., Inoue, S., Kondo, K. and Shimizu, E. (2019) Incidence and Circumstances of Falls among Community-Dwelling Ambulatory Stroke Survivors: A Prospective Study. Geriatrics \& Gerontology International, 19, 240-244. https://doi.org/10.1111/ggi.13594

[34] Walsh, M., Galvin, R. and Horgan, N.F. (2017) Fall-Related Experiences of Stroke Survivors: A Meta-Ethnography. Disability and Rehabilitation, 39, 631-640. https://doi.org/10.3109/09638288.2016.1160445 
[35] Simpson, L.A., Miller, W.C. and Eng, J.J. (2011) Effect of Stroke on Fall Rate, Location and Predictors: A Prospective Comparison of Older Adults with and without Stroke. PLoS One, 6, e19431. https://doi.org/10.1371/journal.pone.0019431

[36] Ng, M.M., Hill, K.D., Batchelor, F. and Burton, E. (2017) Factors Predicting Falls and Mobility Outcomes in Patients with Stroke Returning Home After Rehabilitation Who Are at Risk of Falling. Archives of Physical Medicine and Rehabilitation, 98, 2433-2441. https://doi.org/10.1016/j.apmr.2017.05.018

[37] Wong, J.S., Brooks, D., Inness, E.L. and Mansfield, A. (2016) The Impact of Falls on Motor and Cognitive Recovery after Discharge from In-Patient Stroke Rehabilitation. Journal of Stroke and Cerebrovascular Diseases, 25, 1613-1621. https://doi.org/10.1016/j.jstrokecerebrovasdis.2016.03.017

[38] Agha, S., Levine, I.C. and Novak, A.C. (2020) Determining the Effect of Stair Nosing Shape on Foot Trajectory during Stair Ambulation in Healthy and Post-Stroke Individuals. Applied Ergonomics, 91, Article No. 103304.

https://doi.org/10.1016/j.apergo.2020.103304

[39] Kutzner, I., Heinlein, B., Graichen, F., Bender, A., Rohlmann, A., et al. (2010) Loading of the Knee Joint during Activities of Daily Living Measured in Vivo in Five Subjects. Journal of Biomechanics, 43, 2164-2173.

https://doi.org/10.1016/j.jbiomech.2010.03.046

[40] Forster, A. and Young, J. (1995) Incidence and Consequences of Falls Due to Stroke: A Systematic Inquiry. BMJ, 311, 83-86. https://doi.org/10.1136/bmj.311.6997.83 